\title{
Motion of magnetically charged particles in a magnetically charged stringy black hole spacetime
}

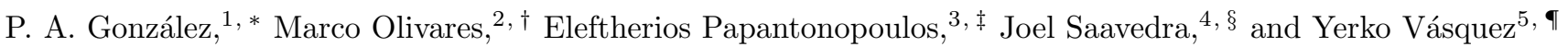 \\ ${ }^{1}$ Facultad de Ingeniería y Ciencias, Universidad Diego Portales, \\ Avenida Ejército Libertador 441, Casilla 298-V, Santiago, Chile. \\ 2 Facultad de Ingeniería y Ciencias, Universidad Diego Portales, \\ Avenida Ejército Libertador 441, Casilla 298-V, Santiago, Chile. \\ ${ }^{3}$ Department of Physics, National Technical University of Athens, Zografou Campus GR 157 73, Athens, Greece. \\ ${ }^{4}$ Instituto de Física, Pontificia Universidad Católica de Valparaíso, Casilla 4950, Valparaíso, Chile. \\ ${ }^{5}$ Departamento de Física y Astronomía, Facultad de Ciencias, Universidad de La Serena, \\ Avenida Cisternas 1200, La Serena, Chile.
}

(Dated: September 21, 2018)

\begin{abstract}
We study the motion of massive particles with electric and magnetic charges in the background of a magnetically charged Garfinkle-Horowitz-Strominger stringy black hole. We solve analytically the equations of motion of the test particles and we describe the orbital motion in terms of the Weierstrass elliptic functions. We find that there are critical values of the magnetic charge of the black hole and the magnetic charge of the test particle which characterize the bound and unbound orbits and we study two observables, the perihelion shift and the Lense-Thirring effect. The trajectories depend on the electric and magnetic charges of the test particle. While the angularmotion depends on the electric charge of the test particle, the $r$ and $t$-motion depends on the mass and the magnetic charge of the test particle.
\end{abstract}

PACS numbers:

\section{Contents}

\section{Introduction}

II. Equations of motion for magnetically charged particles in a magnetically charged stringy black hole

III. General analysis of the equations of motion

A. Analysis of the angular motion ( $\theta$-motion)

B. Analysis of the radial motion ( $r$-motion)

C. Analysis of the angular motion ( $\phi$-motion)

D. Analysis of the time motion ( $t$-motion)

IV. The orbits

V. The observables

\section{Conclusions}

Acknowledgments

References

\footnotetext{
*Electronic address: pablo.gonzalez@udp.cl

${ }^{\dagger}$ Electronic address: marco.olivaresr@mail.udp.cl

$\ddagger$ Electronic address: lpapa@central.ntua.gr

$\S$ Electronic address: joel.saavedra@ucv.cl

๑ Electronic address: yvasquez@userena.cl
} 


\section{INTRODUCTION}

The effective four-dimensional field theory of a heterotic string theory has local black hole solutions which they can have properties very different from those that appear in the black hole solutions of general relativity (GR). A static stringy charged black hole solution of this theory was found in 1, and independently in 2, know as GHS black hole. Thereafter, much research has been performed in the context of the heterotic string theory. In [3] a black hole solution in four dimensions carrying mass, charge and angular momentum was found and also the extremal limit of this black hole solution was discussed. Additionally, this work was further extended in [4 and a general electrically charged, rotating black hole solution in the heterotic string theory compactified on a six-dimensional torus was found. Then it was shown in [5], that given a classical solution of the heterotic string theory other classical solutions can be generated by transforming the original solution. Using this method black string solutions in six dimensions were constructed carrying electric charge, and both, electric and magnetic type antisymmetric tensor gauge field charge. On the other hand, the introduction of the basic aspects of solitons and black holes and duality in string theory [6], stimulated the search of static solutions of electrically and magnetically charged dilaton black holes with various topologies and with the introduction of a cosmological constant [7].

The properties of charged black holes in string theory can be revealed studying the geodesics around these solutions. This is because except the information we get solving the classical equations of motion in the form of Einstein equations we also get information about stringy corrections due to the string coupling which is of the order of Planck scale. The study of null geodesics in the electrically charged GHS black hole was carried out in Refs. [8, 9], and the timelike geodesics were analyzed in [9 13. Additionally, in [14] the gravitational Rutherford scattering and Keplerian orbits were studied in the GHS black hole background.

Magnetically charged black holes have also been studied and their stability was analysed in connection to strong coupling. In the Maxwell-Einstein theory generalized magnetically charged Reissner-Nordström black hole solutions can be found and also in string theory as generalization of electrically charged GHS black hole solutions [1, 2]. An interesting feature of magnetically charged black holes is their connection to magnetic monopoles. In [15] it was shown that a magnetically charged Reissner-Nordström solution develops a classical instability which may lead to a nonsingular magnetic monopole. The magnetic monopoles are hypothetical particles that have not been observed in nature, however, grand unified theories and string theory predict their existence [16]. Also, Dirac showed that the existence of a magnetic monopole in the Universe implies the quantization of the electric charge [17. Additionally, magnetic monopoles appear as regular solutions of the $S U(2)$ Yang-Mills-Higgs theory [18, 19]. A magnetic monopole generated by a gravitational field was discussed in [20].

An important issue in gravitational physics is to know if test particles outside the event horizon of a black hole follow stable circular orbits or not. This information can be provided by studying the geodesics around these black holes. The geodesics of the magnetically charged GHS stringy black hole were analyzed in [9, 21], and it was found that there exists no stable circular orbits outside the event horizon of this stringy black hole for massless test particles. Additionally, the behaviour of null geodesics has been used to calculate the absorption cross section for massless scalar waves at high frequency limit or geometric optic limit, because at high frequency limit the absorption cross section can be approximated by the geometrical cross section of the black hole photon sphere $\sigma \approx \sigma_{\text {geo }}=\pi b_{c}^{2}$, where $b_{c}$ is the impact parameter of the unstable circular orbit of photons. Moreover, in [22, 23. this approximation was improved at the high frequency limit by $\sigma \approx \sigma_{g e o}+\sigma_{o s c}$, where $\sigma_{o s c}$ is a correction involving the geometric characteristics of the null unstable geodesics lying on the photon sphere, such as the orbital period and Lyapunov exponent. This approximation was used recently in [24] to evaluate the absorption cross section of electromagnetic waves at high frequency limit.

In this work we will investigate the timelike geodesics around the magnetically charged GHS black hole. We will show that the geodesic structure for this spacetime will reveal that the motion of charged particles in this magnetically charged space-time will be very different compared to the motion of photons, giving us important information about the structure and properties of this spacetime. For the photons the motion can be studied only in the equatorial plane due to the fact that photons do not carry electric and magnetic charge, therefore they do not feel the Lorentz force. However, in the case of massive particles with electric and magnetic charges because the Lorentz force is perpendicular to the particle four-velocity the motion of the charged particles can not be restricted only on a plane. We will also show in this study, that stringy effects play a crucial role in the behaviour of the orbit, because of the relation of magnetic charge to the string coupling.

We organize the work as follows. In Section II after reviewing in brief the magnetically charged GHS spacetime we present the procedure to obtain the equations of motion of massive particles in the magnetically charged GHS black hole background. In Section III we analyze and solve the equations of motion in terms of the Weierstrass elliptic functions. In Section IV we analyze the orbital motion for a choice of the parameters. In Section $V$ we present in briefly the observables of the perihelion shift and the Lense-Thirring effect. Finally, in Section VI we summarize our results and discuss possible extensions. 


\section{EQUATIONS OF MOTION FOR MAGNETICALLY CHARGED PARTICLES IN A MAGNETICALLY CHARGED STRINGY BLACK HOLE}

In this section we briefly review the magnetically charged black hole in the GHS spacetime (for a review see [25]) and then we present the procedure of obtaining the equations of motion in this spacetime.

The most general action of low energy heterotic string theory is given by

$$
\mathcal{S}=\int d^{D} x \sqrt{-g} e^{-2 \varphi}\left[\Lambda+R+4(\nabla \varphi)^{2}-F_{\mu \nu} F^{\mu \nu}-\frac{1}{12} H_{\mu \nu \rho} H^{\mu \nu \rho}\right],
$$

where the scalar field $\varphi$ is the dilaton field, $F_{\mu \nu}$ is a Maxwell field, and the three form $H_{\mu \nu \rho}$ is related to a two-form potential $B_{\mu \nu}$ and the gauge field $A_{\mu}$ by $H=d B-A \wedge F$ so that $d H=-F \wedge F$. Note that in this action the term $e^{\varphi}$ plays the role of a coupling constant giving the strength of the stringy effects.

If we set $H$ to zero and make the conformal transformation of the metric to rescale $g_{\mu \nu}$ by $e^{-2 \varphi}$ to get a metric with the standard Einstein action

$$
g_{\mu \nu}^{E}=e^{-2 \varphi} g_{\mu \nu}
$$

The action now becomes (with $\Lambda=0$ )

$$
\mathcal{S}=\int d^{4} x \sqrt{-g_{E}}\left(R_{E}-2(\nabla \varphi)^{2}-e^{-2 \varphi} F^{2}\right)
$$

The magnetically charged black hole can be obtained from the electrically charged solution 2 by an electromagnetic duality transformation. From 2.3 , the equation of motion for $F_{\mu \nu}$ is

$$
\nabla_{\mu}\left(e^{-2 \varphi} F^{\mu \nu}\right)=0
$$

This implies $\tilde{F}_{\mu \nu} \equiv e^{-2 \varphi} \frac{1}{2} \epsilon_{\mu \nu}{ }^{\rho \sigma} F_{\rho \sigma}$ is curl free. The equations of motion resulting from the action 2.3 are invariant under $F \rightarrow \tilde{F}, \varphi \rightarrow-\varphi$, and $g_{E} \rightarrow g_{E}$ and the metric of the magnetically charged black hole is given by

$$
d s^{2}=-\frac{f(r)}{a(r)} d t^{2}+\frac{d r^{2}}{f(r) a(r)}+r^{2} d \theta^{2}+r^{2} \sin ^{2} \theta d \phi^{2}
$$

where

$$
f(r) \equiv 1-\frac{2 M}{r}, \quad a(r) \equiv 1-\frac{Q^{2}}{M r},
$$

and the coordinates are defined in the ranges $0<r<\infty,-\infty<t<\infty, 0 \leq \theta<\pi$, and $0 \leq \phi<2 \pi$. $M$ is related to the mass of the spherical object and $Q$ is its magnetic charge. Note that since $\varphi$ changes sign compared to electrically charged solution, the string coupling becomes strong near the singularity for these black holes.

With the aim to study the motion of charged particles around the magnetically charged black hole, first we derive the equations of motion following the standard approach 26. Then, we consider the motion of test particles with mass $m$, electric charge $q$ and magnetic charge $g$ using the Hamilton-Jacobi formalism. In the Appendix we give a detailed account of how the Hamilton-Jacobi formalism is connected with the Euler-Lagrange formalism. The Hamilton-Jacobi equation for the geometry described by metric $g_{\mu \nu}$ for a magnetically and electrically charged test particle is given by

$$
2 \frac{\partial S}{\partial \tau}=g^{\mu \nu}\left(\frac{\partial S}{\partial x^{\mu}}-q A_{\mu}+i g \check{A}_{\mu}\right)\left(\frac{\partial S}{\partial x^{\nu}}-q A_{\nu}+i g \check{A}_{\nu}\right) .
$$

The field strength $F_{\mu \nu}=A_{\nu, \mu}-A_{\mu, \nu}$ and the dual field strength $\check{F}_{\mu \nu}=\check{A}_{\nu, \mu}-\check{A}_{\mu, \nu}$ of the electromagnetic field are induced by the non-vanishing components of the vector potentials $A_{\mu}$ and $\check{A}_{\mu}$

$$
A_{\phi}=-Q \cos \theta, \quad \check{A}_{t}=-\frac{i Q}{r} .
$$

The dual field strength is defined by the antisymmetric Levi-Civita symbol $\varepsilon^{\mu \nu \sigma \tau}$ as $\check{F}^{\mu \nu}=e^{-2 \varphi} \frac{i}{2 \sqrt{g^{d}}} \varepsilon^{\mu \nu \sigma \tau} F_{\sigma \tau}$ with $g^{d}=-\operatorname{det}\left\|g_{\mu \nu}\right\|$. Taking into account the symmetries of the metric under consideration we solve the Hamilton-Jacobi equation using the following ansatz

$$
S=-\frac{1}{2} m^{2} \tau-E t+S_{r}(r)+S_{\theta}(\theta)+L \phi
$$


where $E$ and $L$ are identified as the energy and angular momentum of the test particle. Then, using this ansatz, Eq. (2.6) reads as follows

$$
-m^{2}=-\frac{a(r)}{f(r)}\left[-E+\frac{g Q}{r}\right]^{2}+f(r) a(r)\left(\frac{\partial S_{r}}{\partial r}\right)^{2}+\frac{1}{r^{2}}\left(\frac{\partial S_{\theta}}{\partial \theta}\right)^{2}+\frac{\csc ^{2} \theta(L+q Q \cos \theta)^{2}}{r^{2}} .
$$

We can obtain the following radial equation

$$
-m^{2}=-\frac{a(r)}{f(r)}\left[E-\frac{g Q}{r}\right]^{2}+f(r) a(r)\left(\frac{\partial S_{r}}{\partial r}\right)^{2}+\frac{k}{r^{2}},
$$

and recognizing the Carter separability constant $k$ we obtain the polar equation

$$
k=\left(\frac{\partial S_{\theta}}{\partial \theta}\right)^{2}+\csc ^{2} \theta(L+q Q \cos \theta)^{2} .
$$

Finally, we find formal solutions for the radial and polar components of the action

$$
\begin{gathered}
S_{r}(r, k)=\epsilon \int \sqrt{\left(E-\frac{g Q}{r}\right)^{2}-\frac{f(r)}{a(r)}\left(m^{2}+\frac{k}{r^{2}}\right)} \frac{d r}{f(r)}, \\
S_{\theta}(\theta, k, L)=\epsilon \int \sqrt{k-\csc ^{2} \theta(L+q Q \cos \theta)^{2}} d \theta,
\end{gathered}
$$

where $\epsilon= \pm 1$.

Now, considering $\frac{\delta S}{\delta k}=0, \frac{\delta S}{\delta m^{2}}=0, \frac{\delta S}{\delta E}=0$ and $\frac{\delta S}{\delta L}=0$, and from the Hamilton-Jacobi method, we simplify our study to the following quadrature problem

$$
\begin{gathered}
\int \frac{d \theta}{\sqrt{k-\csc ^{2} \theta(L+q Q \cos \theta)^{2}}}=\int \frac{d r}{r^{2} a(r) \sqrt{\left(E-\frac{g Q}{r}\right)^{2}-\frac{f(r)}{a(r)}\left(m^{2}+\frac{k}{r^{2}}\right)}}, \\
\tau(r)=\epsilon \int \frac{d r}{a(r) \sqrt{\left(E-\frac{g Q}{r}\right)^{2}-\frac{f(r)}{a(r)}\left(m^{2}+\frac{k}{r^{2}}\right)}}, \\
t(r)=\epsilon \int \frac{\left[E-\frac{g Q}{r}\right] d r}{f(r) \sqrt{\left(E-\frac{g Q}{r}\right)^{2}-\frac{f(r)}{a(r)}\left(m^{2}+\frac{k}{r^{2}}\right)}}, \\
\phi(r)=\epsilon \int \frac{\csc ^{2} \theta(L+q Q \cos \theta) d r}{\sqrt{k-c s c^{2} \theta(L+q Q \cos \theta)^{2}}} .
\end{gathered}
$$

Defining the Mino time $\gamma$ as $r^{2} d \gamma=d \tau$, we can express the equations of motion in terms of the new time parameter

$$
\begin{gathered}
\left(\frac{d \theta}{d \gamma}\right)^{2}=k-\csc ^{2} \theta(L+q Q \cos \theta)^{2} \\
\left(\frac{d r}{d \gamma}\right)^{2}=\left(r-Q^{2} / M\right)\left[\left(r-Q^{2} / M\right)(E r-g Q)^{2}-(r-2 M)\left(m^{2} r^{2}+k\right)\right], \\
\frac{d \phi}{d \gamma}=\csc ^{2} \theta(L+q Q \cos \theta), \\
\frac{d t}{d \gamma}=r^{2}\left(\frac{r-Q^{2} / M}{r-2 M}\right)\left[E-\frac{g Q}{r}\right] .
\end{gathered}
$$

In this way we have the equations of motion for our magnetically charged particle moving in the background of stringy magnetically black hole. In the next section will perform a general analysis of the equations of motion. 


\section{GENERAL ANALYSIS OF THE EQUATIONS OF MOTION}

Considering the Hamilton-Jacobi equations (2.18)-2.21), the constants of motion (energy, angular momentum and separation constant), the parameters of the metric and the charges of the test particle we will analyze and solve the equations of motion that characterize the various types of orbits.

\section{A. Analysis of the angular motion ( $\theta$-motion)}

In order to study the $\theta$-motion, we consider the equation of motion (2.18), which can be rewritten using the Mino time as $d \theta / d \gamma=\sqrt{\Theta}$, where the coordinate $\theta$ is a polar angle that can take only positive values. Then,

$$
\Theta=k-\csc ^{2} \theta(L+q Q \cos \theta)^{2} \geq 0
$$

where the separability constant is definite positive. Now, thought the change of variables $\xi=\cos \theta$, equation 2.18 , yields

$$
\frac{d \xi}{d \gamma}=\sqrt{\Theta_{\xi}} ; \quad \text { with } \quad \Theta_{\xi}=k-L^{2}-2 L q Q \xi-\left(k+q^{2} Q^{2}\right) \xi^{2}
$$

The roots of the function $\Theta_{\xi}$ are given by

$$
\begin{aligned}
& \theta_{1}=\cos ^{-1}\left[\frac{L q Q}{k+q^{2} Q^{2}}\left(\sqrt{1+\frac{\left(k-L^{2}\right)\left(k+q^{2} Q^{2}\right)}{L^{2} q^{2} Q^{2}}}-1\right)\right], \\
& \theta_{2}=\cos ^{-1}\left[\frac{L q Q}{k+q^{2} Q^{2}}\left(-\sqrt{1+\frac{\left(k-L^{2}\right)\left(k+q^{2} Q^{2}\right)}{L^{2} q^{2} Q^{2}}}-1\right)\right],
\end{aligned}
$$

which define the cone's angles that confine the movement of the particle. Then, $\gamma(\theta)$ yields

$$
\gamma(\theta)=\frac{1}{\sqrt{k+q^{2} Q^{2}}} \arccos \left[\frac{\left(k+q^{2} Q^{2}\right) \cos \theta+L q Q}{\sqrt{\left(k-L^{2}\right)\left(k+q^{2} Q^{2}\right)+L^{2} q^{2} Q^{2}}}\right],
$$

where we have used that $\gamma_{0}=0$ for $\theta_{0}=\theta_{1}$. Also, the above equation can be inverted, which yields

$$
\theta(\gamma)=\arccos \left[\frac{\sqrt{\left(k-L^{2}\right)\left(k+q^{2} Q^{2}\right)+L^{2} q^{2} Q^{2}} \cos \left(\sqrt{k+q^{2} Q^{2}} \gamma\right)-L q Q}{k+q^{2} Q^{2}}\right]
$$

for $k>L^{2}$.

\section{B. Analysis of the radial motion ( $r$-motion)}

Now, we consider the motion of the particle with respect to the $r$-coordinate. We will focus on equation 2.16 in order to obtain the velocity of the particle $d r / d t$. The condition of turning point $\left(\frac{d r}{d t}\right)_{r=r_{t}}=0$ allows us to define

$$
\left(E-\frac{g Q}{r}\right)^{2}-\frac{f(r)}{a(r)}\left(m^{2}+\frac{k}{r^{2}}\right)=\left(E-V_{-}\right)\left(E-V_{+}\right)
$$

where we can recognize the effective potential for the particle with mass $m$ and magnetic charge $g$ as

$$
V_{ \pm}(r)=\frac{g Q}{r} \pm \sqrt{\frac{f(r)}{a(r)}\left(m^{2}+\frac{k}{r^{2}}\right)}
$$

Since the negative branches have no classical interpretation, they are associated with antiparticles in the framework of quantum field theory [27. We choose the positive branch of the effective potential $V_{\text {eff }}=V_{+} \equiv V$. 
Now, for simplicity we will consider the case where $Q g \leq 0$. Note that $V_{\text {eff }}(r \rightarrow \infty)=m$. So, we plot in Fig. 1 the effective potential for different values of the magnetic charge $Q$ with $g=-0.05, M=1, m=0.1$. We define the the bound and unbound orbits if along the orbits $r$ remains bounded or not. We can observe that there is a critical magnetic charge $Q_{c}$, where the energy of the unstable circular orbit takes the value $m$. For a $Q<Q_{c}$, the effective potential show that all the unbound trajectories $(E \geq m)$ can fall to the horizon or can escape to the infinity. If $Q>Q_{c}$, the maximum value of the effective potential is greater than $m$ and the particles with energy $m<E<E_{c}$ have return points, the magnetic particles located in the right side of the potential that arrive from the infinity have a point of minimum approximation and it is scattered to the infinity which we can call "Magnetic Rutherford scattering". However, for particles located in the left side of the potential have a return point from which plunges to the horizon.

Then, in Fig. 2 we plot the effective potential for different values of the magnetic charge of the test particle $g$ with $M=1, m=0.1, Q=0.75$. Here, we can observe that there a critical value of the magnetic charge of the test particle $g_{c}$ where the energy of the unstable circular orbit takes the value $m$. For a $g>g_{c}$, the effective potential shows that the test particle appears in the scattering zone, while for $g<g_{c}$ does not appear in the scattering zone.

Finally in Fig. 3 we plot the effective potential for different values of the Carter separability constant $k$ with $g=-0.05, M=1, m=0.1$. We can observe that the behaviour is similar to the first case, that is, there a critical value of the Carter separability constant $k_{c}$, where the energy of the unstable circular orbit takes the value $m$. For a $k<k_{c}$, the effective potential shows that all the unbound trajectories $(E \geq m)$ can fall to the horizon or can escape to the infinity. If $k>k_{c}$, the maximum value of the effective potential is greater than $m$ and the particles with energy $m<E<E_{c}$ have a return points, the magnetic particles located in the right side of the potential that arrive from the infinity have a point of minimum approximation and it is scattered to the infinity which we have called "Magnetic Rutherford scattering". However, for particles located in the left side of the potential have a return point from which plunge to the horizon.

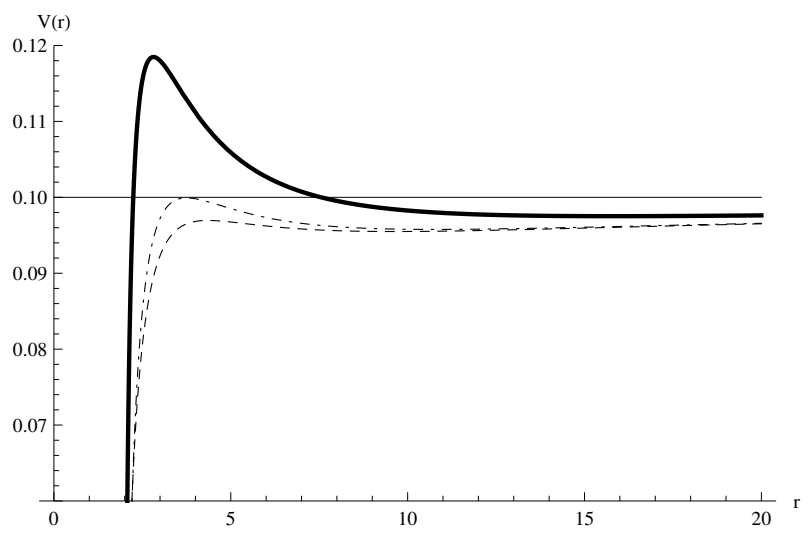

FIG. 1: Effective potential for $g=-0.05, M=1, m=0.1$, and different values of the magnetic charge $Q=0$ (dashed line), $Q=Q_{c} \approx 1.02$ (dot-dashed line), $Q=1.3$ (thick line) and $E=m$ (thin horizontal line). 


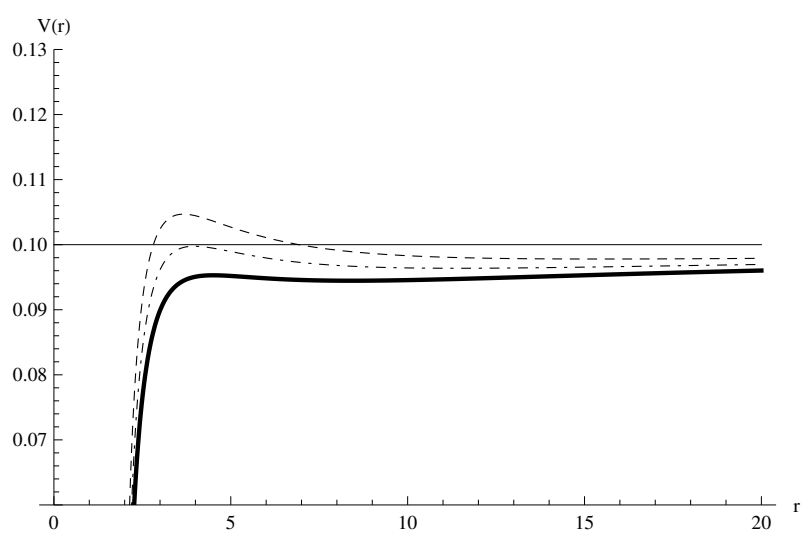

FIG. 2: Effective potential for $M=1, m=0.1, Q=0.75$ and different values of the magnetic charge of the test particle $g=0$ (dashed line), $g=g_{c} \approx-0.025$ (dot-dashed line), $g=-0.05$ (thick line) and $E=m$ (thin horizontal line).

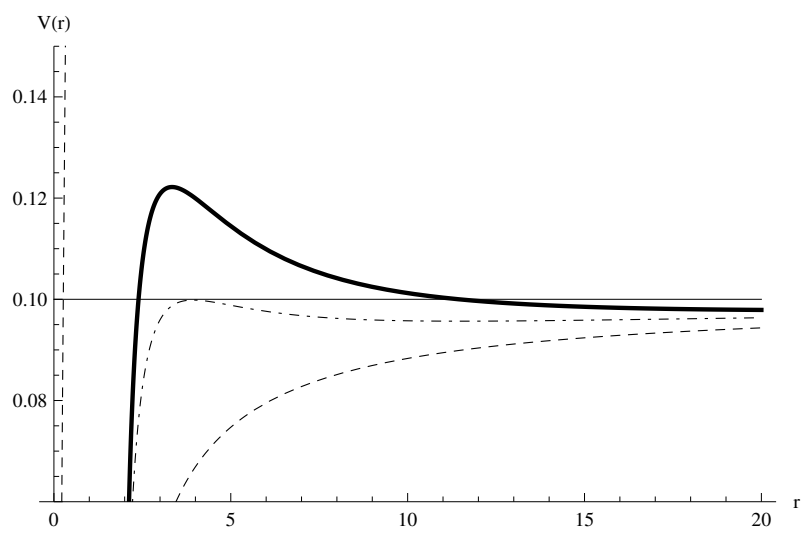

FIG. 3: Effective potential for $g=-0.05, M=1, m=0.1$ and different values of the Carter separability constant $k=0$ (dashed line), $k=k_{c} \approx 0.168$ (dot-dashed line), $k=0.3$ (thick line) and $E=m$ (thin horizontal line).

Let us rewrite the radial equation 2.19 as

$$
\left(\frac{d r}{d \gamma}\right)^{2}=\left(r-\frac{Q^{2}}{M}\right) R(r),
$$

where we define

$$
R(r) \equiv\left(r-Q^{2} / M\right)(E r-g Q)^{2}-(r-2 M)\left(m^{2} r^{2}+k\right),
$$

now this function can be written as the characteristic polynomial

$$
R(r)=a_{3} r^{3}+a_{2} r^{2}+a_{1} r+a_{0}
$$

where

$$
\begin{array}{ll}
a_{0}=2 k M-\frac{g^{2} Q^{4}}{M}, & a_{1}=-k+g^{2} Q^{2}+\frac{2 g Q^{3} E^{2}}{M} \\
a_{2}=2 m^{2} M-2 g Q E-\frac{Q^{2} E^{2}}{M}, & a_{3}=E^{2}-m^{2} .
\end{array}
$$

Now, by performing a change of variables

$$
U(r)=\frac{1}{4(r-\alpha)}+\frac{\beta_{1}}{12},
$$


we obtain

$$
r(\gamma)=\left(\frac{Q^{2}}{M}\right)+\frac{1}{4 \wp\left(\omega_{0} \mp \sqrt{b_{0}} \gamma ; g_{2}, g_{3}\right)-\beta_{1} / 3},
$$

where $\wp\left(x ; g_{2}, g_{3}\right)$ is the $\wp$-Weierstrass elliptic function, with the Weierstrass invariant given by

$$
g_{2}=\frac{\beta_{1}^{2}}{12}-\frac{\beta_{2}}{4}, \quad g_{3}=\frac{\beta_{1} \beta_{2}}{48}-\frac{\beta_{1}^{3}}{216}-\beta_{3}, \quad \text { and } \quad \omega_{0}=\wp^{-1}\left[\frac{1}{4\left(r_{0}-Q^{2} / M\right)}+\frac{\beta_{1}}{12}\right],
$$

where

$$
\beta_{1}=\frac{b_{1}}{b_{0}}, \quad \beta_{2}=\frac{b_{2}}{b_{0}}, \quad \beta_{3}=\frac{a_{3}}{b_{0}},
$$

and $r_{0}$ corresponds to an initial arbitrary distance and

$$
\begin{aligned}
& b_{0}=a_{0}+a_{1}\left(\frac{Q^{2}}{M}\right)+a_{2}\left(\frac{Q^{2}}{M}\right)^{2}+a_{3}\left(\frac{Q^{2}}{M}\right)^{3}, \\
& b_{1}=a_{1}+2 a_{2}\left(\frac{Q^{2}}{M}\right)+3 a_{3}\left(\frac{Q^{2}}{M}\right)^{2}, \\
& b_{2}=a_{2}+3 a_{3}\left(\frac{Q^{2}}{M}\right) .
\end{aligned}
$$

\section{Analysis of the angular motion ( $\phi$-motion)}

In order to obtain the $\phi$-motion, we consider Eq. 2.18 and Eq. 2.20 which allow us to write

$$
\phi(\theta)=\int_{\theta_{1}}^{\theta} \frac{(L+q Q \cos \theta) d \theta}{\sin ^{2} \theta \sqrt{\Theta}}
$$

which yields

$$
\begin{aligned}
\phi(\theta)= & \frac{1}{2} \arccos \left(\frac{\left(k-L q Q+q^{2} Q^{2}\right)(1+\cos \theta)-(L-q Q)^{2}}{(1+\cos \theta) \sqrt{k\left(k-L^{2}+q^{2} Q^{2}\right)}}\right)+ \\
& \frac{1}{2}\left(\arcsin \left(\frac{\left(k+L q Q+q^{2} Q^{2}\right)(1-\cos \theta)-(L+q Q)^{2}}{(1-\cos \theta) \sqrt{k\left(k-L^{2}+q^{2} Q^{2}\right)}}\right)+\frac{\pi}{2}\right),
\end{aligned}
$$

where $L>q Q$, and we have used as initial condition $\phi\left(\theta_{1}\right)=0$ for simplicity. Then, it is possible to obtain $\phi(\gamma)$ by replacing $\theta(\gamma)$ in the above expression.

\section{Analysis of the time motion ( $t$-motion)}

Now, in order to describe the time motion we rewrite Eq. 2.16 as

$$
t(r)=\int_{r_{0}}^{r} r^{2}\left(\frac{r-\alpha}{r-2 M}\right)\left(E-\frac{g Q}{r}\right) \frac{d r}{\sqrt{(r-\alpha) R(r)}},
$$

and using the same change of variables that we have used in the radial motion (3.13), we obtain the following solution

$$
t(r)=A_{1}\left[F_{1}(r)-F_{1}\left(r_{0}\right)\right]+A_{2}\left[F_{2}(r)-F_{2}\left(r_{0}\right)\right]+A[F(r)],
$$

where

$$
A_{1}=\frac{-g Q+E(2 \alpha+\beta)}{4 \sqrt{b_{0}}}, \quad A_{2}=\frac{g Q(\alpha+\beta)-E(\alpha+\beta)^{2}}{4 \beta \sqrt{b_{0}}}, \quad A=E
$$




$$
F_{i}(r)=\frac{1}{\wp^{\prime}\left(\Omega_{i}\right)}\left[\zeta\left(\Omega_{i}\right) \wp^{-1}(U)+\ln \left|\frac{\sigma\left[\wp^{-1}(U)-\Omega_{i}\right]}{\sigma\left[\wp^{-1}(U)+\Omega_{i}\right]}\right|\right],
$$

with

$$
\Omega_{1}=\wp^{-1}\left(\frac{\beta_{1}}{12}\right), \quad \Omega_{2}=\wp^{-1}\left(\frac{3+\beta \beta_{1}}{12 \beta}\right)
$$

and

$$
\left.F(r)=A_{0}\left(\Pi-\Pi_{0}\right)-\sum_{i=1}^{2} \frac{1}{\left(\wp^{\prime}\left[\omega_{i}\right]\right)^{2}}\left[\zeta\left[\Pi-\omega_{i}\right]-\zeta\left[\Pi_{0}-\omega_{i}\right]+\frac{\wp^{\prime \prime}\left[\omega_{i}\right]}{\wp^{\prime}\left[\omega_{i}\right]} \ln \mid \frac{\sigma\left[\Pi-\omega_{i}\right]}{\sigma\left[\Pi_{0}-\omega_{i}\right]}\right]\right],
$$

with

$$
\Pi \equiv \wp^{-1}(U) \quad \text { and } \quad \frac{\beta_{1}}{12}=\frac{1}{\left(\wp\left[\omega_{1}\right]-\frac{\beta_{1}}{12}\right)^{2}}=\frac{1}{\left(\wp\left[\omega_{2}\right]-\frac{\beta_{1}}{12}\right)^{2}}
$$

and

$$
A_{0}=-\sum_{i=1}^{2}\left[\frac{\wp\left[\omega_{i}\right]}{\left(\wp^{\prime}\left[\omega_{i}\right]\right)^{2}}+\frac{\wp^{\prime \prime}\left[\omega_{i}\right] \zeta\left[\omega_{i}\right]}{\left(\wp^{\prime}\left[\omega_{i}\right]\right)^{3}}\right]
$$

where we have used $\Pi_{0}=\wp^{-1}\left[U\left(r_{0}\right)\right]$.

\section{THE ORBITS}

In this section we analyze the different kind of orbits that the test particles follow in the magnetically charged stringy black hole for the following parameters $m=0.1, q=0.05, g=-0.05, M=1$ and $Q=0.75$. The potential is shown in Fig. 4 , where we observe the two possible cases, one of them is the potential for bound orbits $(E<m)$ and the other one is the potential for unbound orbits $(E \geq m)$. The orbits of the first kind are the relativistic analogues of the Keplerian orbits to which they tend in the Newtonian limit. The orbits of the second kind have no Newtonian analogues [26].

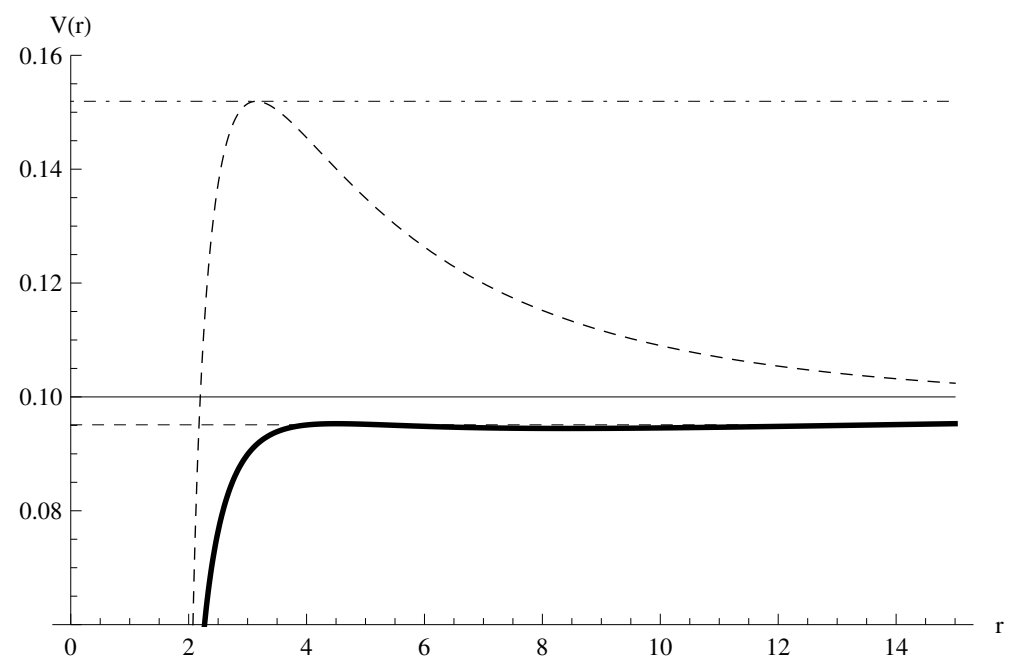

FIG. 4: Effective potential for bound orbits $k=0,14$ (thick line), unbound orbit $k=0,5$ (dashed line), $E=0.0951, E \approx 0.1519$, and $E=m$ with $g=-0.05, Q=0.75, M=1, m=0.1$. 
In order to obtain a full description of the radial motion of charged particles, we study separately the two possible cases. For bound orbits, in Fig. 5 we show the behaviour of the orbit for a charged particle, that oscillate between the periastro and apoastro distance, this first kind of orbit corresponds to a planetary orbit. Also, we show the projection of the orbit onto the $x-z$-plane. Then, in Fig. 6 we show the orbit of a particle with the same energy that the previous case, but it is localized to left of the potential. Also, we show the projection of the orbit onto the $x-z$-plane it is an orbit of the first kind. Then, in Fig. 7 we show the behaviour of critical orbits of first kind with $E=0.0953$, we can observe that the trajectory starts at a certain aphelion distance and approaches the circle, asymptotically, by spiralling around it an infinite number of times. Also, we show the projection of the orbit onto the $x-z$-plane.

In Fig. 8 we show the behaviour of critical orbits of second kind with the same energy that the previous case, but the particle is localized to left of the potential. Also, we show the projection of the orbit onto the $x-z$-plane. On the other hand, for unbound orbits in Fig. 9 we show the trajectory of a particle that arrive from infinity with $E=0.1519$, and we show the projection of the orbit onto the $x-z$-plane. It is an orbit of the first kind, due to the fact that the orbit is analogue to the hyperbolic orbit of the Newtonian theory [26].
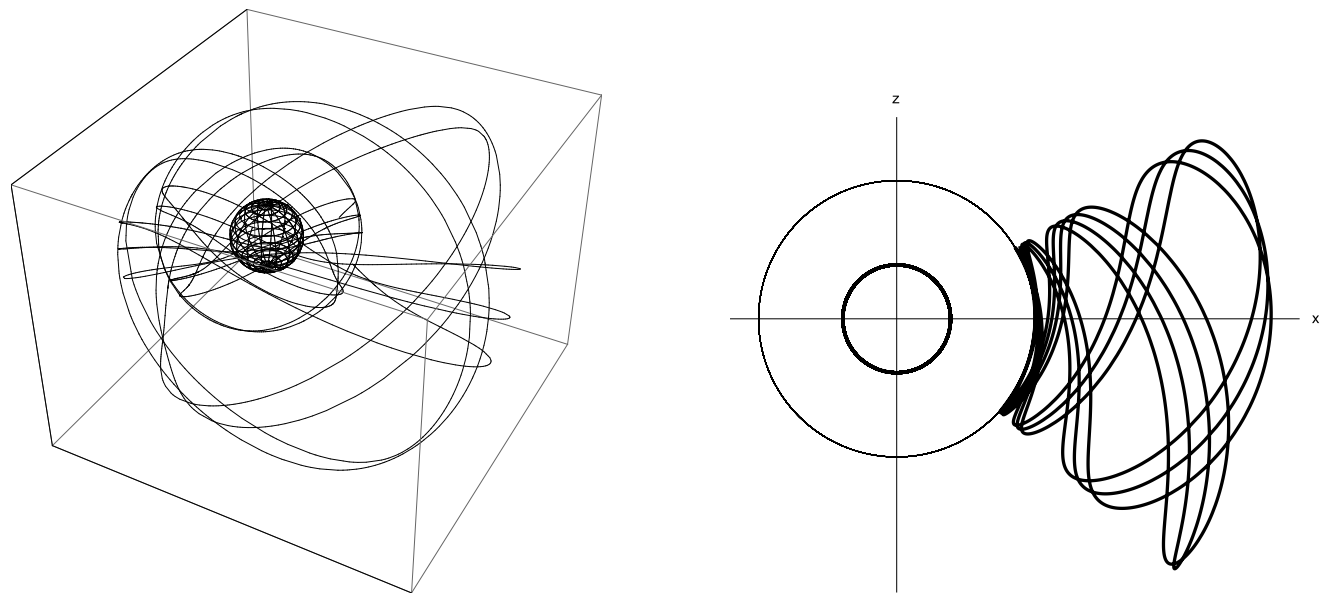

FIG. 5: Planetary orbit with $k=0.14, L=0.3$ and $E=0.0951$. Left figure for three-dimensional motion, where the central circle correspond to the event horizon, and right figure is the projection of the orbit onto the $x-z$-plane, where the small circle corresponds to the event horizon and the other one corresponds to the periastro distance.
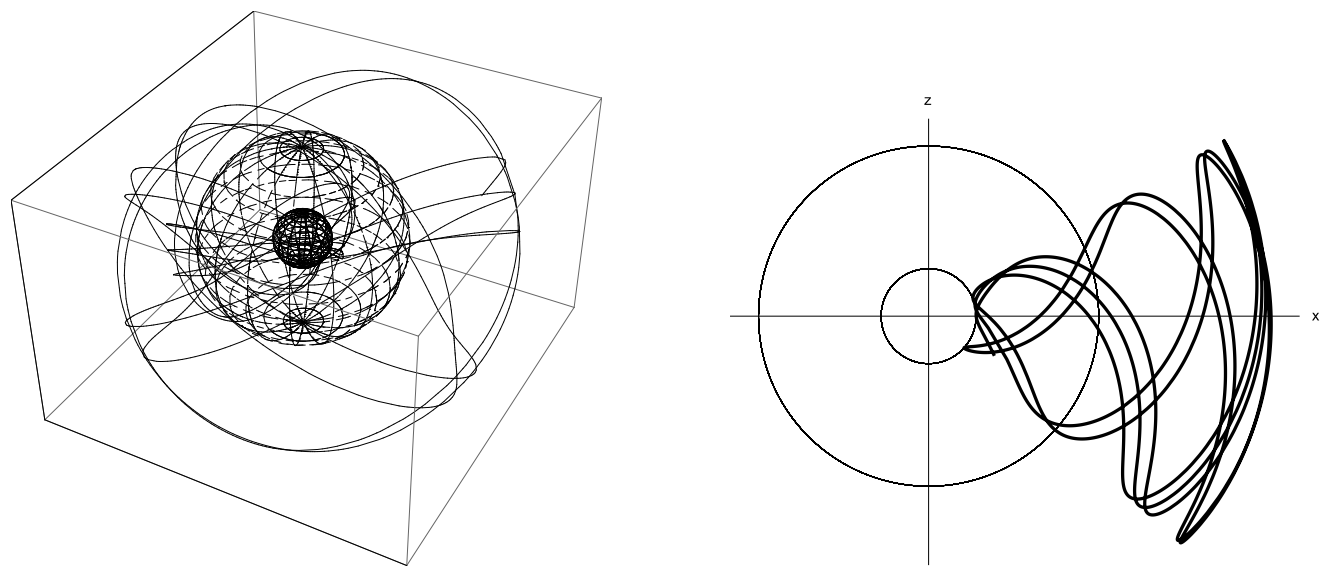

FIG. 6: Bound orbits of second kind with $k=0.14, L=0.3$ and $E=0.0951$. Left figure for three-dimensional motion, where the central circle corresponds to a return point inside the horizon (by simplicity we choose $Q^{2} / M$, as return point), and right figure is the projection of the orbit onto the $x-z$-plane, where the small circle corresponds to the return point and the other one corresponds to event horizon. Note that the trajectory has a physical meaning outside the horizon. 

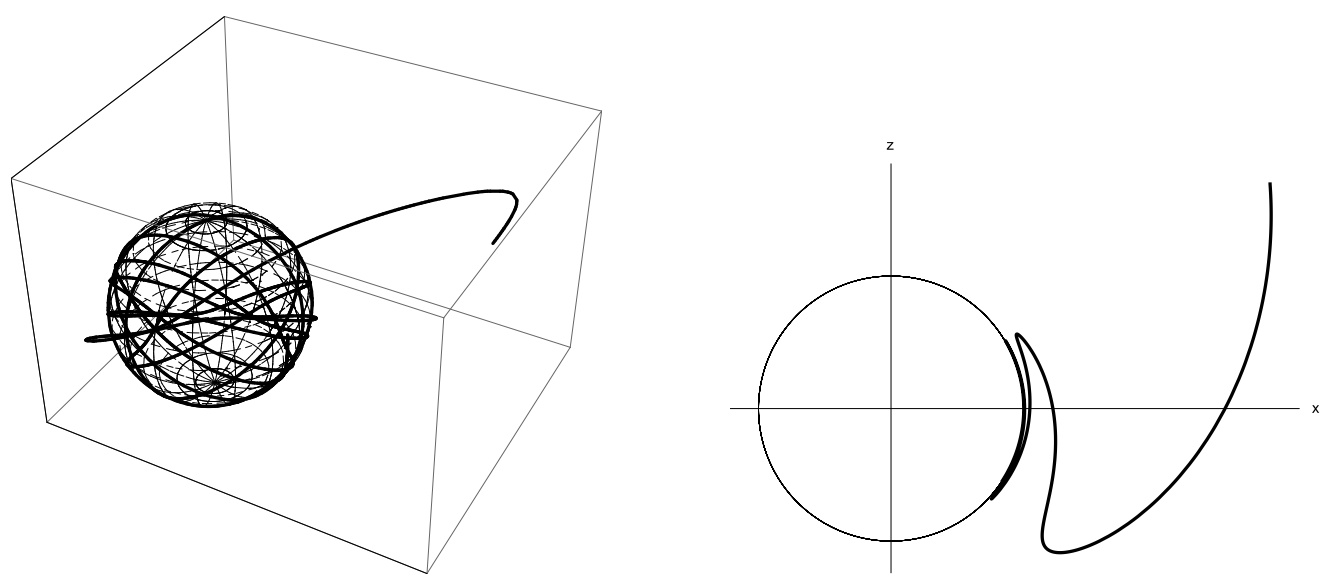

FIG. 7: Critical orbits of first kind with $k=0.14, L=0.3$ and $E=0.0953$. Left figure for three-dimensional motion, and right figure is the projection of the orbit onto the $x-z$-plane. The circle corresponds to the unstable circular orbit.
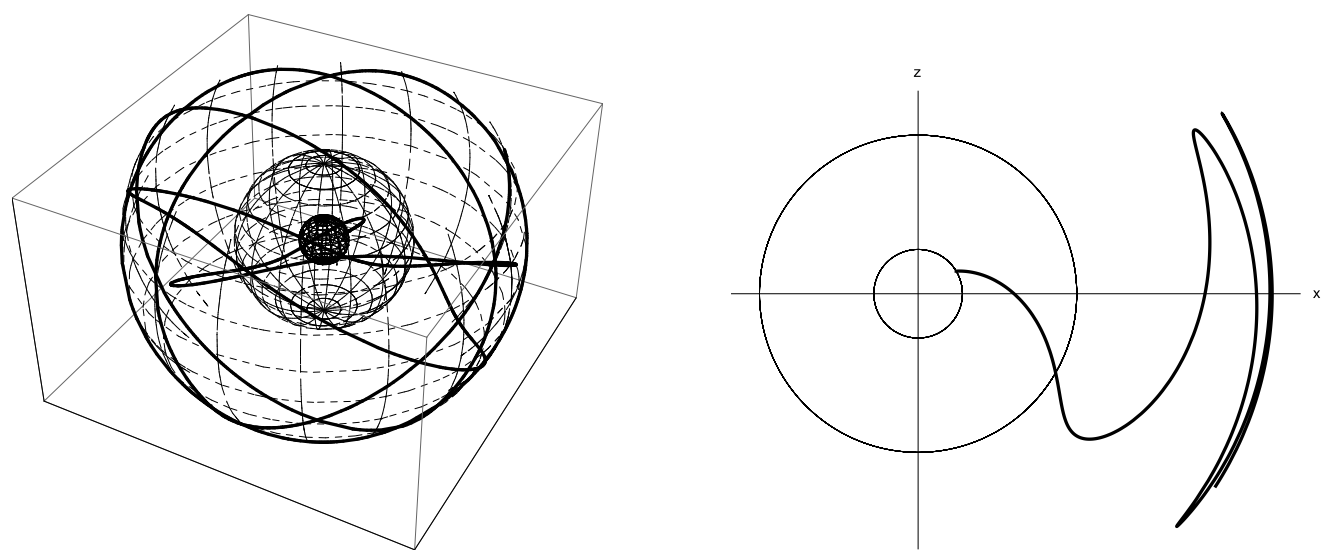

FIG. 8: Critical orbits of second kind with $k=0.14, L=0.3$ and $E=0.0953$. Left figure for three-dimensional motion, where the central circle corresponds to a return point inside the horizon (by simplicity we choose $Q^{2} / M$, as return point), and right figure is the projection of the orbit onto the $x-z$-plane, where the small circle corresponds to the return point and the other one corresponds to event horizon. Note that the trajectory has a physical meaning outside the horizon.

\section{THE OBSERVABLES}

In this section following [28] we calculate some possible observables. However, as our work is about massive particles we will study the perihelion shift and the Lense-Thirring effect. So, we consider here bound orbits, like the planetary orbits, these orbits precess between the aphelion distance, $r_{A}$, and the perihelion distance, $r_{P}$. The $r$-motion is periodic with a period given by

$$
\omega_{r}=2 \gamma\left(r_{A}\right)=\frac{2}{\kappa_{p}}\left(\wp^{-1}\left[\frac{1}{4\left(r_{P}-Q^{2} / M\right)}+\frac{\beta_{1}}{12}\right]-\wp^{-1}\left[\frac{1}{4\left(r_{A}-Q^{2} / M\right)}+\frac{\beta_{1}}{12}\right]\right) .
$$

The corresponding orbital frequency is $2 \pi / \omega_{r}$. On the other hand, the period of the $\theta$-motion is given by

$$
\omega_{\theta}=2 \gamma\left(\theta_{2}\right)=\frac{2 \pi}{\sqrt{k+q^{2} Q^{2}}}
$$

and the corresponding frequency by $2 \pi / \omega_{\theta}$.

The secular accumulation rates of the angle $\phi$ and the time $t$ are given by

$$
Y_{\phi}=\frac{2}{\omega_{\theta}} \phi\left(\theta_{2}\right)=\sqrt{k+q^{2} Q^{2}}
$$



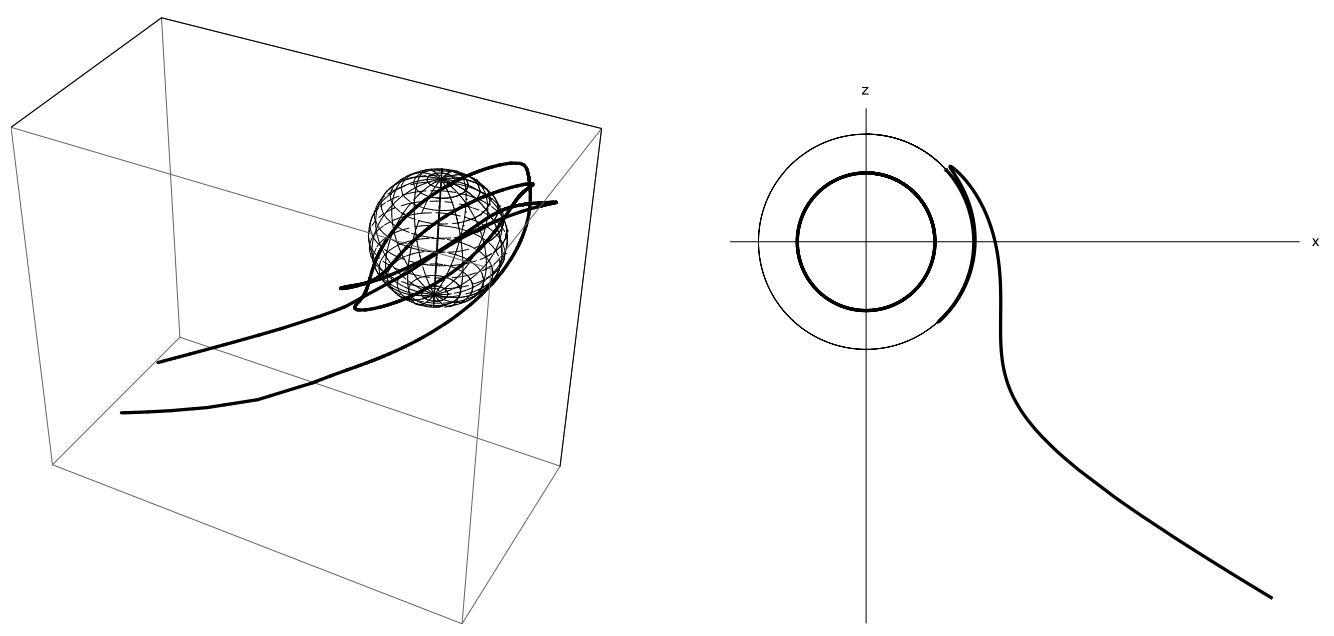

FIG. 9: Trajectory for a particle that arrive from infinity (Magnetic Rutherford scattering) with $k=0.5, L=0.3$ and $E=0.1519$. Left figure for three-dimensional motion, and right figure is the projection of the orbit onto the $x-z$-plane. The small circle correspond to the event horizon and the other one corresponds to the closest approach distance.

$$
\Gamma=\frac{2}{\omega_{r}} t\left(r_{A}\right)=\frac{2}{\omega_{r}}\left(A_{1}\left[F_{1}\left(r_{A}\right)-F_{1}\left(r_{P}\right)\right]+A_{2}\left[F_{2}\left(r_{A}\right)-F_{2}\left(r_{P}\right)\right]+A\left[F\left(r_{A}\right)\right]\right),
$$

and the orbital frequencies $\Omega_{r}, \Omega_{\theta}$ and $\Omega_{\phi}$ read

$$
\Omega_{r}=\frac{2 \pi}{\omega_{r}} \frac{1}{\Gamma}, \quad \Omega_{\theta}=\frac{2 \pi}{\omega_{\theta}} \frac{1}{\Gamma}, \quad \Omega_{\phi}=\frac{Y_{\phi}}{\Gamma} .
$$

The perihelion shift and the Lense-Thirring effects are defined as differences between these orbital frequencies

$$
\begin{gathered}
\Delta_{\text {Perihelion }}=\Omega_{\phi}-\Omega_{r}=\frac{1}{\Gamma}\left(\sqrt{k+q^{2} Q^{2}}-\frac{2 \pi}{\omega_{r}}\right), \\
\Delta_{\text {Lense-Thirring }}=\Omega_{\phi}-\Omega_{\theta}=0 .
\end{gathered}
$$

Therefore, we can see that there is no Lense-Thirring effect, due to the fact that the orbital frequencies $\Omega_{\phi}$ and $\Omega_{\theta}$ coincide as it was found in [28, 29]. This happens because in the Lense-Thirring effect three rotations are involved, one of them is the rotation of the gravitating body, the other is the rotation of the test body around its own axis, and the last one is the rotation of the axis of rotation of the test body. However, in the case that we have analyzed these rotations are not present.

\section{CONCLUSIONS}

In this work we studied the motion of massive particles with electric and magnetic charges in the background of the the magnetically charged Garfinkle-Horowitz-Strominger black hole. We solved analytically the equations of motion for the test particles in terms of the Weierstrass $\wp, \sigma$ and $\zeta$ elliptic functions. We found that, the radial $r$-motion and time $t$-motion depends on the mass and the magnetic charge of the test particle, while angular-motion depends only on the electric charge of the test particle. We note that in the case of a test particle moving in the background of electrically charged GHS black hole, its radial motion depends on the parameters that define the test particle, that is, the mass and electric charge of the test particle [14.

Analyzing the effective potential, we found that bound orbits exist for $E<m$, while for $E \geq m$ the orbits are unbound, where $m$ and $E$ are the mass and energy of the test particle. In analogy to the Gravitational Rutherford scattering observed in [14] we have observed the dispersion between the magnetic charge of the black hole and the magnetic charge of the test particle, which we have called Magnetic Rutherford scattering. Finally, we have studied two observables, the perihelion shift and the Lense-Thirring effects. The perihelion shift depends on electric and magnetic charges of the test particle and there is no Lense-Thirring effect due to the fact that the rotations involved in the Lense-Thirring effect are not present in the case analyzed. 
The behaviour of the radial motion of the test particle except its mass, it dependents also on two crucial parameters, the magnetic charge of the black hole $\mathrm{Q}$ and the magnetic charge $\mathrm{g}$ of the test particle. We found in the Section IIIB that the stability of the radial motion of the test particle, if it crosses the horizon or if it goes to infinity depends on a critical value of $Q_{c}$ and $g_{c}$. However, the magnetic charge Q of the GHS black hole is related to the strength of the string coupling through the relation $e^{-2 \varphi}=1-\frac{Q^{2}}{M r}$. Therefore, the string coupling will effect the behaviour of radial motion of the test particle.

It would be interesting to see if the magnetically charged GHS black hole can radiate its charge and what is the role of the string coupling in this effect. However, the test particle we considered in this work does not have its own dynamics to trigger such an effect. We plan to study in the lines of [30, 31] the superradiance effect of a magnetically charged scalar wave scattered off the magnetically charged GHS black hole.

\section{Acknowledgments}

This work was partially funded by Comisión Nacional de Ciencias y Tecnología through FONDECYT Grants 11140674 (PAG) and by the Dirección de Investigación y Desarrollo de la Universidad de La Serena (Y.V.). P. A. G. acknowledges the hospitality of the Universidad de La Serena and National Technical University of Athens and E. P. and Y. V. acknowledge the hospitality of the Universidad Diego Portales where part of this work was carried out.

\section{Appendix A: Hamilton-Jacobi formalism}

In this Appendix we show how the Hamilton-Jacobi formalism is connected to the Euler-Lagrange formalism. From the Lagrangian formulation you can construct the Hamiltonian and then perform a canonical transformation in order to obtain the Hamilton-Jacobi equation. In the Lagrangian formalism one define a Lagrangian from the metric

$$
2 \mathcal{L}=g_{\mu \nu} \dot{x}^{\mu} \dot{x}^{\nu}=-m,
$$

where $\dot{x}^{\mu}=\frac{d x^{\mu}}{d \tau}$ and $\tau$ is an affine parameter, which can be the proper time for massive particles. The Hamiltonian is given by

$$
\mathcal{H}=p_{\mu} \dot{x}^{\mu}-\mathcal{L}=\frac{1}{2} g^{\mu \nu} p_{\mu} p_{\nu},
$$

where $d x^{\mu} / d \tau$ was expressed in terms of the conjugate momentum and the coordinates. Now, one can perform a canonical transformation, from the coordinates of the phase space $x^{\mu}, p_{\nu}$ where the Hamiltonian is $H$ to the coordinates $X^{\mu}, P_{\nu}$ where the Hamiltonian is $K$

$$
p_{\mu} d x^{\mu}-H d \tau=P_{\mu} d X^{\mu}-K d \tau+d F,
$$

where $F$ is a function of the phase space coordinates and $\tau$. Considering the transformations

$$
p_{\mu}=p_{\mu}\left(x^{\alpha}, P_{\nu}, \tau\right), X^{\mu}=X^{\mu}\left(x^{\nu}, P_{\alpha}, \tau\right)
$$

and integrating by parts the term $P_{\mu} d X^{\mu}$, we get

$$
p_{\mu} d x^{\mu}-H d \tau=-X^{\mu} d P_{\mu}-K d \tau+d\left(F+X^{\mu} P_{\mu}\right) .
$$

Defining the generating function $S$ of the canonical transformation as

$$
S\left(x^{\sigma}, P_{\mu}, \tau\right)=F+X^{\mu} P_{\mu},
$$

we obtain

$$
d S=\frac{\partial S}{\partial x^{\mu}} d x^{\mu}+\frac{\partial S}{\partial P_{\mu}} d P_{\mu}+\frac{\partial S}{\partial \tau} d \tau
$$

Then equation $(\mathrm{A} 5)$ gives

$$
\left(p_{\mu}-\frac{\partial S}{\partial x^{\mu}}\right) d x^{\mu}-\left(\mathcal{H}+\frac{\partial S}{\partial \tau}\right) d \tau=-\left(X^{\mu}-\frac{\partial S}{\partial P_{\mu}}\right) d P_{\mu}-K d \tau .
$$


From this equation we get

$$
\begin{gathered}
p_{\mu}=\frac{\partial S}{\partial x^{\mu}}, \\
X^{\mu}=\frac{\partial S}{\partial P_{\mu}}, \\
K=\mathcal{H}\left(x^{\mu}, p_{\sigma}, \tau\right)+\frac{\partial S}{\partial \tau} .
\end{gathered}
$$

Setting the new Hamiltonian $K$ to zero we obtain a coordinate transformation for $S$

$$
\mathcal{H}\left(x^{\mu}, \frac{\partial S}{\partial x^{\sigma}}, \tau\right)+\frac{\partial S}{\partial \tau}=0
$$

Then using the Hamiltonian A2 we finally get

$$
\frac{1}{2} g^{\mu \nu} \frac{\partial S}{\partial x^{\mu}} \frac{\partial S}{\partial x^{\nu}}+\frac{\partial S}{\partial \tau}=0
$$

In the case of the presence of electromagnetic fields the Hamilton-Jacobi equation is trivially modified.

[1] G. W. Gibbons and K. i. Maeda, "Black Holes and Membranes in Higher Dimensional Theories with Dilaton Fields," Nucl. Phys. B 298, 741 (1988).

[2] D. Garfinkle, G. T. Horowitz and A. Strominger, "Charged black holes in string theory," Phys. Rev. D 43, 3140 (1991) Erratum: [Phys. Rev. D 45, 3888 (1992)].

[3] A. Sen, "Rotating charged black hole solution in heterotic string theory," Phys. Rev. Lett. 69, 1006 (1992) hep-th/9204046].

[4] A. Sen, "Black hole solutions in heterotic string theory on a torus," Nucl. Phys. B 440, 421 (1995) hep-th/9411187.

[5] S. F. Hassan and A. Sen, "Twisting classical solutions in heterotic string theory," Nucl. Phys. B 375, 103 (1992) hepth/9109038.

[6] R. R. Khuri, "Solitons, black holes and duality in string theory," Nucl. Phys. Proc. Suppl. 61A, 99 (1998) hep-th/9704110.

[7] C. J. Gao and S. N. Zhang, "Topological black holes in dilaton gravity theory," Phys. Lett. B 612, 127 (2005).

[8] S. Fernando, "Null Geodesics of Charged Black Holes in String Theory," Phys. Rev. D 85, 024033 (2012) arXiv:1109.0254 [hep-th]].

[9] S. Soroushfar, R. Saffari and E. Sahami, Phys. Rev. D 94, no. 2, 024010 (2016) arXiv:1601.03143 [gr-qc]].

[10] T. Maki and K. Shiraishi, "Motion of test particles around a charged dilatonic black hole," Class. Quant. Grav. 11, 227 (1994).

[11] M. Olivares and J. R. Villanueva, "Massive neutral particles on heterotic string theory," Eur. Phys. J. C 73, 2659 (2013) arXiv:1311.4236 [gr-qc]].

[12] C. Blaga, "Circular time-like geodesics around a charged spherically symmetric dilaton black hole," Applications Math. 22, 41 (2013) arXiv:1406.7421 [gr-qc]].

[13] C. Blaga, "Timelike geodesics around a charged spherically symmetric dilaton black hole," Serb. Astron. J. 190, 41 (2015) arXiv:1407.1504 [gr-qc]].

[14] J. R. Villanueva and M. Olivares, "Gravitational Rutherford scattering and Keplerian orbits for electrically charged bodies in heterotic string theory," Eur. Phys. J. C 75, no. 11, 562 (2015), arXiv:1510.08340 [gr-qc]].

[15] K. M. Lee, V. P. Nair and E. J. Weinberg, "A Classical instability of Reissner-Nordstrom solutions and the fate of magnetically charged black holes," Phys. Rev. Lett. 68, 1100 (1992) hep-th/9111045.

[16] X. G. Wen and E. Witten, "Electric and Magnetic Charges in Superstring Models," Nucl. Phys. B 261, 651 (1985).

[17] P. A. M. Dirac, "Quantized Singularities in the Electromagnetic Field," Proc. Roy. Soc. Lond. A 133, 60 (1931).

[18] G. 't Hooft, "Magnetic Monopoles in Unified Gauge Theories," Nucl. Phys. B 79, 276 (1974).

[19] A. M. Polyakov, "Particle Spectrum in the Quantum Field Theory," JETP Lett. 20, 194 (1974) [Pisma Zh. Eksp. Teor. Fiz. 20, 430 (1974)].

[20] M. Barriola and A. Vilenkin, "Gravitational Field of a Global Monopole," Phys. Rev. Lett. 63, 341 (1989).

[21] R. S. Kuniyal, R. Uniyal, H. Nandan and K. D. Purohit, "Null Geodesics in a Magnetically Charged Stringy Black Hole Spacetime," arXiv:1509.05131 [gr-qc].

[22] Y. Decanini, G. Esposito-Farese and A. Folacci, "Universality of high-energy absorption cross sections for black holes," Phys. Rev. D 83, 044032 (2011) arXiv:1101.0781 [gr-qc]].

[23] Y. Decanini, A. Folacci and B. Raffaelli, "Fine structure of high-energy absorption cross sections for black holes," Class. Quant. Grav. 28, 175021 (2011) arXiv:1104.3285 [gr-qc]]. 
[24] S. Fernando and A. Manning, "Electromagnetic perturbations of a de Sitter black hole in massive gravity," arXiv:1701.01983 [gr-qc].

[25] G. T. Horowitz, "The dark side of string theory: Black holes and black strings.," In *Trieste 1992, Proceedings, String theory and quantum gravity ' $92 *$ 55-99 hep-th/9210119.

[26] Chandrasekhar S.: The Mathematical Theory of Black Holes. Oxford University Press, New York (1983).

[27] N. Deruelle and R. Ruffini, "Quantum and classical relativistic energy states in stationary geometries," Phys. Lett. 52B, 437 (1974).

[28] V. Kagramanova, J. Kunz, E. Hackmann and C. Lammerzahl, "Analytic treatment of complete and incomplete geodesics in Taub-NUT space-times," Phys. Rev. D 81, 124044 (2010) arXiv:1002.4342 [gr-qc]].

[29] S. Grunau and V. Kagramanova, "Geodesics of electrically and magnetically charged test particles in the ReissnerNordstróm space-time: analytical solutions," Phys. Rev. D 83, 044009 (2011) arXiv:1011.5399 [gr-qc]].

[30] P. A. González, E. Papantonopoulos, J. Saavedra and Y. Vásquez, "Superradiant Instability of Near Extremal and Extremal Four-Dimensional Charged Hairy Black Hole in anti-de Sitter Spacetime," arXiv:1702.00439 [gr-qc].

[31] T. Kolyvaris and E. Papantonopoulos, "Superradiant Amplification of a Scalar Wave Coupled Kinematically to Curvature Scattered off a Reissner-Nordström Black Hole," arXiv:1702.04618 [gr-qc]. 